Proc. XII Int. School on Theoretical Physics - Symmetry and Structural Properties of Condensed Matter

\title{
Interaction Independent Quantum Probing
}

\author{
G. SARBicki ${ }^{a, *}$, M. Tukiainen ${ }^{b}$, H. Lyyra ${ }^{b}$ And S. MAniscalCo ${ }^{b, c}$ \\ ${ }^{a}$ Institute of Physics, Nicolaus Copernicus University, Grudziądzka 5/7, 87-100 Torun, Poland \\ ${ }^{b}$ Turku Centre for Quantum Physics, Department of Physics and Astronomy, University of Turku, \\ FI-20014 Turun yliopisto, Finland \\ ${ }^{c}$ Centre for Quantum Engineering, Department of Applied Physics, School of Science, Aalto University, \\ P.O. Box 11000, FIN-00076 Aalto, Finland
}

For an open quantum system we assume that we are able to set the system's environment temperature. We fix the time interval and let the system (further referred as the probing system) to evolve during this time in two different temperatures. We make a process tomography of the resulting dynamics (quantum channels $\varepsilon_{1}, \varepsilon_{2}$ related to the temperatures $T_{1}$ and $T_{2}$ respectively). We calculate then the values of $\alpha$-fidelities for the pair of channels. We derive an inequality between the experimental data and the partition function of environment (hence the spectrum of the environment). If the inequality is not satisfied, it implies that our assumption about the spectrum of the environment is wrong. Notice that there is no dependence on the interaction terms neither on the Hamiltonian of the probing system. We show the power of this method in the following example. Consider a two-level atom passing the one-mode vacuum. We do not know the Hamiltonian of the atom (the probing system) neither the interaction mechanism. We would like to determine the frequency of the vacuum. We will show that wide range of frequencies are forbidden by the inequality.

DOI: 10.12693/APhysPolA.132.103

PACS/topics: quantum entropies, quantum programming, open quantum systems

\section{Introduction}

The Shannon entropy of a state $S(p)=-\sum_{i} p_{i} \log _{2} p_{i}$ is a measure of information contained in the state. The Kulback-Leiber divergence $S(p \| q)=\sum_{i} p_{i} \log _{2}\left(p_{i} / q_{i}\right)$ is a (non-symmetrical) measure of distance between two states, namely it tells how surprised we are by the measuring result if we assume a distribution $p$, but the source is producing symbols with respect to a distribution $q$ (or how much more bits on average a codeword for a symbol will have if the code is optimised with respect to the distribution $q$, but the source produces symbols due to $p)$.

There is one-parameter family of entropies generalising the Shannon entropy called the Rényi entropies defined as:

$$
H_{\alpha}(p)=\frac{1}{1-\alpha} \log _{2} \sum_{i} p_{i}^{\alpha},
$$

where $\alpha \in(0, \infty) \backslash 0$. One has $H_{\alpha \rightarrow 1}=S$. The corresponding divergences are defined as $H_{\alpha}(p \| q)=$ $\frac{1}{\alpha-1} \log _{2} \sum_{i: q_{i}>0} p_{i}^{\alpha} q_{i}^{1-\alpha}$ and are called the Rényi divergencies of order $\alpha$.

The Rényi entropies are easily generalised to the quantum case: $H_{\alpha}(\varrho)=\frac{1}{1-\alpha} \operatorname{Tr} \varrho^{\alpha}$. It approaches the von Neumann entropy when $\alpha \rightarrow 1$. Due to noncommutativity of quantum states, there are at least two ways of generalisation of the Rényi divergences to the quantum case: the non-symmetric version $H_{\alpha}(\varrho \| \sigma)=$

*corresponding author; e-mail: gniewko@fizyka.umk.pl $\frac{1}{\alpha-1} \log _{2} \operatorname{Tr}\left(\varrho^{\alpha} \sigma^{1-\alpha}\right) \quad[1]$ and the symmetrised one $H_{\alpha}(\varrho \| \sigma)=\frac{1}{\alpha-1} \log _{2} \operatorname{Tr}\left(\left(\sigma^{\frac{1-\alpha}{2 \alpha}} \varrho \sigma^{\frac{1-\alpha}{2 \alpha}}\right)^{\alpha}\right)[2]$. We have focused on the second one.

It is useful to consider closely related quantites called $\alpha$-fidelities defined as $F_{\alpha}(\varrho \| \sigma)=\operatorname{Tr}\left(\left(\sigma^{\frac{1-\alpha}{2 \alpha}} \varrho \sigma^{\frac{1-\alpha}{2 \alpha}}\right)^{\alpha}\right)$. Observe that $F_{1 / 2}$ is the usual (Uhlmann) fidelity and this fact stays behind our choice of the symmetrised quantum Rényi divergence.

The Rényi divergence for $\alpha \in(0,1)$ has the following properties:

1. $H_{\alpha}(\varrho \| \sigma) \geq 0$, equality if $\sigma=\varrho$.

2. $H_{\alpha}\left(\varrho_{1} \otimes \xi_{1} \| \varrho_{2} \otimes \xi_{2}\right)=H_{\alpha}\left(\varrho_{1} \| \varrho_{2}\right)+H_{\alpha}\left(\xi_{1} \| \xi_{2}\right)$.

3. $H_{\alpha}(\mathcal{U}(\varrho) \| \mathcal{U}(\sigma))=H_{\alpha}(\varrho \| \sigma)$ for any unitary channel $\mathcal{U}$

Additionally for $\alpha \in\left[\frac{1}{2}, 1\right)$ one has the data processing inequality:

4. $H_{\alpha}(\varepsilon(\varrho) \| \varepsilon(\sigma)) \leq H_{\alpha}(\varrho \| \sigma)$ for any quantum channel $\varepsilon$.

\section{Quantum programming inequalities and fidelities of channels}

Any pair of quantum channels on an open quantum system can be realised as a partial trace of a unitary channel on the bigger closed system

$$
\varepsilon_{i}(\varrho)=\operatorname{Tr}_{B}\left(\mathcal{U}\left(\varrho \otimes \xi_{i}\right)\right) .
$$

We say then that a channel $\varepsilon_{i}$ is induced by a state $\xi_{i}$. 
Using the above properties of the Rényi divergences one can prove the following inequality involving channels and their inducing states:

$$
H_{\alpha}\left(\varepsilon_{1}\left(\varrho_{1}\right) \| \varepsilon_{2}\left(\varrho_{2}\right)\right) \leq H_{\alpha}\left(\xi_{1} \| \xi_{2}\right)+H_{\alpha}\left(\varrho_{1} \| \varrho_{2}\right)
$$

for $\alpha \in\left(\frac{1}{2}, 1\right)$. One can get rid of the arguments of the channels

$$
\begin{aligned}
& H_{\alpha}\left(\xi_{1}, \xi_{2}\right) \geq \\
& \quad \inf \varrho_{1}, \varrho_{2}\left(H_{\alpha}\left(\varepsilon_{1}\left(\varrho_{1}\right) \| \varepsilon_{2}\left(\varrho_{2}\right)\right)-H_{\alpha}\left(\varrho_{1} \| \varrho_{2}\right)\right),
\end{aligned}
$$

of using the $\alpha$-fidelities

$$
F_{\alpha}\left(\xi_{1}, \xi_{2}\right) \leq \inf \varrho_{1}, \varrho_{2}\left(\frac{F_{\alpha}\left(\varepsilon_{1}\left(\varrho_{1}\right), \varepsilon_{2}\left(\varrho_{2}\right)\right)}{F_{\alpha}\left(\varrho_{1}, \varrho_{2}\right)}\right) .
$$

the right hand side (RHS) will be the definition of fidelity of channels $\varepsilon_{1}, \varepsilon_{2}$ and the above inequality says, that the $\alpha$-fidelity of two channels is always greater or equal to the $\alpha$-fidelity of their inducing states.

A programmable quantum computer is a device realising a constant unitary operation on two registers: the data register and the program word register. Tracing out the program register, the device performs a channel on the data register, induced by the state of the program register. The inequalities (3) relates the $\alpha$-fidelity of channels to the the $\alpha$-fidelity of their inducing (programming) states. That is why we call them the quantum programming inequalities.

In particular, the $\alpha$-fidelity of two unitary channels is 1 if they differ by a phase factor, otherwise 0 . This implies that their programming states have to be orthogonal, and using the quantum programming register of $N$ qubits one can program only $2^{N}$ unitary operations on the data register, hence the universal quantum computer (implementing all possible unitary gates) is not possible (see [3]).

\section{Probing the environment}

If one couples a quantum system to an environment and wait for a time $t$, a state of the system is affected by a quantum channel induced by the Gibbs state of the environment. Assume that we are able to couple a probing system to the same environment prepared in two different inverse temperatures $\beta_{1}$ and $\beta_{2}$. Two Gibbs states commute and hence calculation of LHS of (3) is very simple. One gets

$$
\begin{aligned}
& \ln \left(Z\left(\alpha \beta_{1}+(1-\alpha) \beta_{2}, H_{E}\right)\right) \\
& \quad-\alpha \ln \left(Z\left(\beta_{1}, H_{E}\right)\right)-(1-\alpha) \ln \left(Z\left(\beta_{2}, H_{E}\right)\right) \\
& \quad \leq \inf _{t \geq 0}\left\{\begin{array}{l}
\ln \left(\mathcal{F}_{\alpha}\left(\varepsilon_{2}^{(t)}, \varepsilon_{1}^{(t)}\right)\right), \text { for } \alpha \in\left(0, \frac{1}{2}\right) \\
\ln \left(\mathcal{F}_{\alpha}\left(\varepsilon_{1}^{(t)}, \varepsilon_{2}^{(t)}\right)\right), \text { for } \alpha \in\left[\frac{1}{2}, 1\right)
\end{array} .\right.
\end{aligned}
$$

According to our assumptions and predictions about the Hamiltonian of the environment, we calculate the left hand side (LHS). We fix the time $t$ and perform full quantum process tomography of $\varepsilon_{1}$ and $\varepsilon_{2}$ and then calculate $\mathcal{F}_{\alpha}\left(\varepsilon_{1}^{(t)}, \varepsilon_{2}^{(t)}\right)$ via numerical optimisation from the experimental data. If the inequality is not satisfied for any value of $\alpha$, then our assumption about the environment is wrong. Notice that no knowledge about the interaction term in Hamiltonian (neither about the Hamiltonian of the probing system) is necessary.

\section{Example}

Assume that the system of interest is a 2-level atom in a multi-mode vacuum

$$
\begin{aligned}
& H=H_{S}+H_{E}+H_{I}= \\
& \quad \frac{\hbar \omega_{0}}{2} \sigma_{z}+\sum_{k} \hbar \omega_{k}\left(b_{k}^{\dagger} b_{k}+\frac{1}{2} I\right)+\sum_{k} \sigma_{z}\left(g_{k} b_{k}^{\dagger}+g_{k}^{*} b_{k}\right),
\end{aligned}
$$

this model is exactly solvable (up to Born, Markov and RW approximately, see [4]) and one gets

$$
\varepsilon^{(t)}(\varrho)=\left(\begin{array}{cc}
\varrho_{00} & \Gamma(t) \varrho_{01} \\
\Gamma(t) \varrho_{10} & \varrho_{11}
\end{array}\right),
$$

where

$$
\Gamma(t)=\exp \left[-\sum_{k} \frac{4\left|g_{k}\right|^{2}}{\hbar \omega_{k}^{2}} \operatorname{coth}\left(\frac{1}{2} \beta \hbar \omega_{k}\right)\left[1-\cos \left(\omega_{k} t\right)\right]\right] .
$$

It is possible to determine the value of the RHS

$$
\begin{aligned}
& \mathcal{F}_{\alpha}\left(\varepsilon_{1}^{(t)}, \varepsilon_{2}^{(t)}\right)=\left(\frac{1+\Gamma_{2}(t)}{2}\right)^{1-\alpha}\left(\frac{1+\Gamma_{1}(t)}{2}\right)^{\alpha} \\
& +\left(\frac{1-\Gamma_{2}(t)}{2}\right)^{1-\alpha}\left(\frac{1-\Gamma_{1}(t)}{2}\right)^{\alpha} .
\end{aligned}
$$

Let us assume now that the true environment is a onmode vacuum of an unknown frequency $\omega_{1}$. We assume a value (unknown) of $\omega$ and we calculate the LHS of (4):

$$
\begin{aligned}
& -\log _{2}\left(1-\exp \left(-\left(\alpha \beta_{1}+(1-\alpha) \beta_{2}\right) \omega\right)\right) \\
& \quad+\alpha \log _{2}\left(1-\exp \left(-\beta_{1} \omega\right)\right) \\
& \quad+(1-\alpha) \log _{2}\left(1-\exp \left(-\beta_{2} \omega\right)\right) .
\end{aligned}
$$

For the RHS calculated (measured) for $\beta_{1} \hbar=2, \beta_{2} \hbar=$ $20, g_{1} / \sqrt{\hbar}=0.3$ and $\omega_{1}=1$, we calculate the LHS for 3 assumed values of $\omega: 3,3.1$, and 3.5 (see Fig. 1). The limit value is $\approx 3.1$ (red). The value 3.5 (cyan) is forbidden.

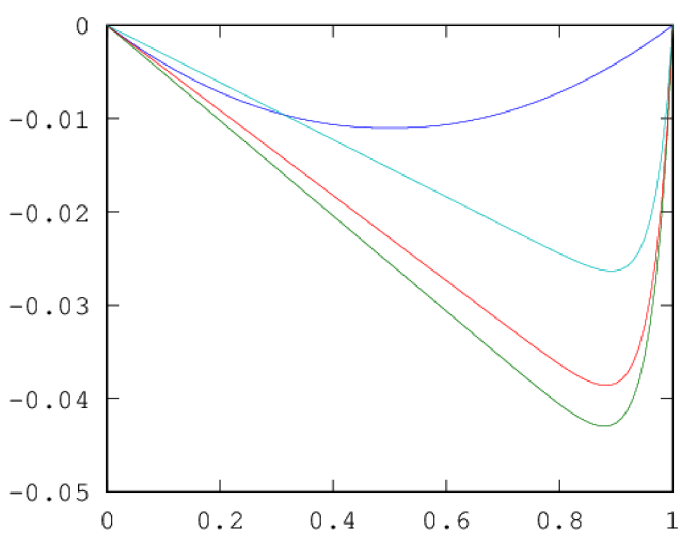

Fig. 1. LHS vs. $\alpha$ for $\omega=1$ (blue), $\omega=1$ (green), $\omega=3.1$ (red), $\omega=3.5$ (cyan). 


\section{Conclusions}

The presented method is general and applies to any open quantum system interacting with environment. It lets us to falsify the assumptions about the spectrum of the environment or to find the restrictions on the set of free parameters in a considered model of systemenvironment interaction. In contrary to all known methods of probing the environment (up to our best knowledge), our method does not require any knowledge about the mechanism of interaction. For details, see [5].

\section{References}

[1] D. Petz, Rep. Math. Phys. 23, 57 (1986).

[2] M.M. Wilde, A. Winter, D. Yang, Commun. Math. Phys. 331C, 593 (2014).

[3] M.A. Nielsen, I.L. Chuang, Quantum Computation and Quantum Information, Cambridge University Press, 2000.

[4] H.P. Breuer, F. Petruccione, The Theory of Open Quantum Systems, Vol. 1, Oxford University Press, New York 2007, p. 227.

[5] M. Tukiainen, H. Lyyra, G. Sarbicki, S. Maniscalco, Fidelity of Dynamical Maps, arXiv:1609.00482. 